\title{
Clinical Characteristics And Health Care Resources In Patients Treated With Oral Anticoagulants: Evidences From Italian Administrative Databases
}

This article was published in the following Dove Press journal: Vascular Health and Risk Management

\section{Degli Esposti ${ }^{1}$ \\ $M$ Andretta $^{2}$ \\ G Di Pasquale ${ }^{3}$ \\ M Gambera ${ }^{4}$ \\ S Saragoni ${ }^{1}$ \\ V Perrone' \\ S Buda $\mathbb{D}^{\prime}$}

'CliCon S.r.l. Health, Economics \& Outcomes Research, Ravenna, Italy; ${ }^{2}$ Local Pharmaceutical Service, Verona Local Health Authority, Verona, Italy; ${ }^{3}$ Department of Cardiology, Maggiore Hospital, Bologna, Italy; ${ }^{4}$ Local Pharmaceutical Service, Bergamo Local Health Authority, Bergamo, Italy
Correspondence: $\mathrm{V}$ Perrone CliCon S.r.l, Health, Economics and Outcomes Research, Via Salara, 36, Ravenna 48100, Italy

Tel +39054438393

Fax +390544212699

Email valentina.perrone@clicon.it
Objectives: 1) To evaluate anticoagulation treatment patterns and health care resource use in adult patients with a discharge diagnosis of non-valvular atrial fibrillation (NVAF) in an Italian real-world setting and 2) to describe the characteristics of NVAF patients in relation to treatment.

Design: A retrospective cohort study in a "real-world" setting.

Setting: Data were analysed by integrating administrative databases that included approximately 2,000,000 individuals assisted by the National Health System from two Italian Local Health Units.

Participants: All adult patients with at least one hospital discharge or $\geq 2$ outpatient visits with a diagnosis code for NVAF from 1/01/2011 to 31/12/2015 were included.

Main outcome measures: Anticoagulation treatment patterns, health care resource use and major bleeding events that occurred during the follow-up period were evaluated.

Results: 32,863 NVAF patients were included, of whom 7,831 had at least one prescription of oral anticoagulants. Among them, 6,876 patients were vitamin K antagonists (VKA) users and 955 were non-vitamin $\mathrm{K}$ antagonist oral anticoagulant (NOAC) users at index date (ID). During the follow-up period, the use of antiplatelet drugs was higher among VKA-naïve users than the NOAC-naïve users. Among NOAC users, 76.1\% showed an adherence level $\geq 80 \%$ during follow-up. The rate of bleeding events resulted higher for VKA patients compared to NOAC patients. The unadjusted incidence rate was 10.46 per 1000 personyear for VKA patients and 4.55 per 1,000 person-years for NOAC patients. The overall annual cost (in term of drugs, hospitalisations and outpatient specialist services) was $€ 5,156.13$ for VKA and $€ 4,630.57$ for NOAC.

Conclusion: This unselected cohort study, on NVAF patients being prescribed oral anticoagulants, highlights that VKA was largely prescribed and the great majority of patients on NOACs were adherent to treatment. Most of the OAC patients still received antiplatelet agents in combination, and in NOAC patients, we registered a lower number of bleeding events compared with VKA.

Keywords: non-valvular atrial fibrillation, vitamin $\mathrm{K}$ antagonists, non-vitamin $\mathrm{K}$ antagonist oral anticoagulants, anticoagulation treatment patterns, real-world setting

\section{Introduction}

Non-valvular atrial fibrillation (NVAF), the most common type of cardiac arrhythmia worldwide, affects approximately $1-2 \%$ of the general population and is associated with a substantial health care and economic burden. ${ }^{1}$ Most importantly, NVAF was correlated to an increased risk of cardiovascular mortality and 
hospitalisation. The presence of this arrhythmia is an independent risk factor for stroke/thromboembolism and death, with an estimated 5-fold higher risk. ${ }^{1,2}$

Currently, vitamin $\mathrm{K}$ antagonists (VKA) and four nonvitamin $\mathrm{K}$ antagonist oral anticoagulants (NOACs) are available for stroke prevention in patients with NVAF in Italy: dabigatran etexilato (available from June 2013), rivaroxaban (available from September 2013), apixaban (available from January 2014) and edoxaban (available from January 2016). ${ }^{1,3}$ The efficacy of these drugs has been proven in pivotal clinical studies; moreover, these trials have demonstrated a favourable safety profile of NOACs compared to VKA. ${ }^{4-7}$ However, little information is available on actual health care resource use among patients treated with oral anticoagulants (OACs) in clinical settings in Italy. $^{8}$

The study aims to fill this information gap, to increase the awareness on the economic burden associated with treatment of patients with NVAF in Italy and provide information of the actual local health care resource allocation in individual regions. The key intended audience for the study is commissioners for Health Care Service in individual Italian Local Health Units (LHU).

To gain a comprehensive understanding, from an Italian practice-based perspective, on the current use of NVAF treatments in Italy, we conducted a study with the following two main objectives: 1) To evaluate anticoagulation treatment patterns and health care resource use in adult patients with a discharge diagnosis of NVAF, in an unselected Italian real-world setting and 2) to describe the characteristics of patients affected by NVAF in relation to treatment.

\section{Methods}

\section{Data Sources}

This study was conducted using administrative and clinical databases (DBs) of 2 Italian LHUs geographically distributed throughout the national territory (Verona, Veneto; Bergamo, Lombardy), for a total number of about 2,000,000 healthassisted individuals.

The following databases were used in this study: Beneficiaries' DB, which contains patient demographic data; Pharmaceuticals DB, which includes information for each medication prescription, such as the prescribing physician's number and the Anatomical-Therapeutic-Chemical (ATC) code of the prescribed drug; Hospitalisation DB, which includes information on discharge for each hospitalisation, in particular the date of admission and discharge, main and secondary diagnosis, coded according to the International Classification of Diseases, Ninth Revision, Clinical Modification (ICD-9-CM) and Diagnosis-Related Group (DRG) reimbursement rate; Diagnostic tests and specialist visits $D B$, which includes all information about outpatient specialist services and finally Laboratory DB, which includes all information about type of laboratory tests, date of test and results.

The data were extracted by each LHU and their databases were anonymised in full compliance with the Italian code of protection of personal data (Legislative Decree, 196/03). To guarantee patient privacy, each subject was assigned an anonymous patient number. The patient code in each database permitted electronic linking across all databases. No identifiers related to patients were provided to the researchers. All the results of the analyses were produced as aggregated summaries, which are not possible to assign, either directly or indirectly, to the individual patients. Informed consent is not required for encrypted retrospective information. This study was notified to the local ethics committee of each participating LHU, according to the Italian regulation on observational studies, and the LHU Ethics Committees approved the study.

\section{Cohort Definition}

This was an observational retrospective cohort study, which included all patients aged $\geq 18$ years with at least one $(\geq 1)$ hospital discharge or $\geq 2$ outpatient visits with an ICD-9-CM diagnosis code for NVAF from 1 January 2011 to 31 December 2015 (identification period).

Patients were considered treated if they had at least one prescription of VKA (warfarin) or NOAC (rivaroxaban, apixaban and dabigatran) during the identification period. The date of the first prescription during the identification period represented the "index date" (ID) for each patient. Only cohort of treated patients during the identification period and continuous enrolment in the LHU 12 months prior to the ID were considered for the first and second objectives in the analysis. Patients enrolled in the analysis were observed from the ID until 31 December 2015 (follow-up period) and their clinical characteristics were assessed at baseline and 12 months prior to the ID (characterisation period).

Exclusion criteria were considered as follows: diagnosis of valvular atrial fibrillation, cardiac surgery (coronary artery bypass graft or other cardiac surgery, pericarditis, myocarditis, venous thromboembolism), diagnosis of 
valvular heart disease, hyperthyroidism, pregnancy and valve replacement, registered during the characterisation period or within 6 months before NVAF diagnosis. According to their first prescription of OAC (VKA or NOACs) during the enrolment period, the patients were stratified into the following four main categories: 1) "Prevalent OAC users": patients treated with VKA at ID and previously (pre-ID) treated with OAC (VKA); 2) "NOAC naïve users, OAC experienced": patients treated with NOAC at ID and previously (pre-ID) treated with OAC (VKA); 3) "NOAC naïve users, OAC naïve": patients treated with NOAC at ID and previously (pre-ID) not treated with OAC (VKA); 4) "VKA naïve users, OAC naïve": patients treated with VKA and previously (pre-ID) not treated with OAC (VKA).

\section{Definitions And Study Variables}

Data on baseline characteristics, including demographics, hospital admissions, prescribed drugs and comorbidity, during the characterisation period, were collected. We identified the following diseases by using ICD-9-CM codes retrieved from hospital admissions (both primary and secondary diagnoses collected in the database were considered): hypertension, chronic kidney disease, chronic obstructive and pulmonary disease, cirrhosis/hepatitis or other disorders of liver, coagulopathy, coronary artery disease, diabetes mellitus, heart failure, stroke, intracranical bleeding, major bleeding, acute myocardial infarction, paraplegia or hemiplegia, psychiatric disorders and intellectual disabilities. Previous pharmacological treatments were analysed only when at least 2 prescriptions were reported, as not to include occasional prescriptions.

The major bleeding events (gastrointestinal, nontraumatic intracranial, traumatic intracranial and from other sites) that occurred during the follow-up period were evaluated. The analysis on bleeding events was only among patients with the same therapeutic strategy during the follow-up period. All ATC code and ICD-9-CM codes used for all analysis are reported in the Supplementary File.

\section{Treatment Adherence}

Adherence to NOACs was measured as the proportion of days covered (PDC) over a 365-day period. PDC was computed as the actual number of days of NOAC therapy supplied divided by 365, and multiplied by 100 . Days' supply was calculated based on the quantity dispensed and dosage unit per day indicated to NVAF patients: dabigatran dosages: $150 \mathrm{mg}$ or $110 \mathrm{mg}$ 2/twice a day; apixaban dosage
$5 \mathrm{mg} /$ twice a day; rivaroxaban dosage $20 \mathrm{mg} /$ once a day. Adherence to treatment analysis was performed only in NOAC patients with the same class of therapy and followed-up for at least 12 months.

\section{Cost Analysis}

A cost analysis was conducted with the perspective of the Italian National Health System (NHS) among adult patients (aged $\geq 18$ years) with at least one prescription of VKA (Warfarin) or NOAC (rivaroxaban, apixaban and dabigatran) from 1 July 2013 to 30 June 2015. Only cohort of treated patients during the identification period and with at least 1 year of follow-up period was considered to estimate health care costs.

All costs are reported in Euros $(€)$. Drug costs were evaluated using the Italian NHS purchase price. Hospitalisation costs were determined using the DRG (diagnosis-related group) tariffs. The mean annual health care costs per patient were evaluated based on total resource consumption (in term of drugs, hospitalisations and outpatient specialist services) during the follow-up period. DRG tariffs represent the reimbursement levels of the NHS to health care providers. The outpatient service costs were defined according to the tariffs applied by the evaluated regions. The mean annual total health care costs were estimated separately in VKA and NOAC users, respectively. For cost analysis in VKA users, a standard dose of $2.5 \mathrm{mg} /$ day was assumed. ${ }^{9}$

\section{Statistical Analysis}

Descriptive analyses are presented in the paper. Continuous variables were reported as mean and $\pm \mathrm{SD}$, whereas categorical variables were expressed as numbers and percentages. According to the Italian Regulation on protection of personal data (D.Lgs. 196/2003 ${ }^{10}$ ), results referring to a number of patients $<4$ cannot be presented as they are potentially reconductable to single individuals. In this case, results were reported as NI (not issuable).

Univariate analyses ( $\chi^{2}$ test and Student $t$-test) were used to compare baseline characteristics of VKA-naïve users and NOAC-naïve users.

All statistical analyses were performed using STATA $\mathrm{SE}$, version 12.0. Data management was carried out using Microsoft SQL Server 2012.

\section{Patient And Public Involvement}

Nor patients or public were involved in the design and conduction of the study. 


\section{Results}

Over a population of about 2,000,000 individuals registered, we enrolled 32,863 patients with a diagnosis of NVAF (about $1.6 \%$ of the health-assisted individuals). Figure 1 shows the details of the study's inclusion and exclusion criteria. Of the 32,863 identified patients in the databases, 7,831 had at least one prescription of OAC and on which the analyses were conducted.

Among patients treated with OAC agents, 6,876 had a prescription of a VKA and 955 had a prescription of a NOAC at baseline (ID). The distribution of the study population stratified by treatment assignment 1 year before the ID is shown in Figure 2. Among patients treated with VKA at ID, 50.0\% received VKA during the characterisation period (pre-ID) (Figure 2), whereas, among NOAC users, $4.6 \%$ patients were previously treated with a VKA (Figure 2). As the enrolment start date for the present study was earlier than the marketing authorisation date of the first NOAC, none of our cohorts included patients previously treated with NOAC.

Among the NOAC users, the percentage of patients treated with high doses (150 mg for dabigatran; $20 \mathrm{mg}$ for rivaroxaban; $5 \mathrm{mg}$ for apixaban) or with low doses (110 $\mathrm{mg}$ for dabigatran; $10 \mathrm{mg}$ and $15 \mathrm{mg}$ for rivaroxaban;

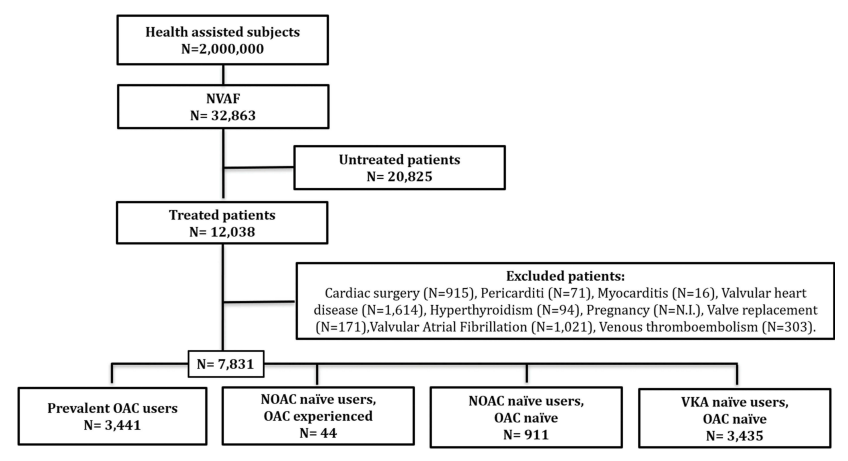

Figure I Flow chart of cohort definition.

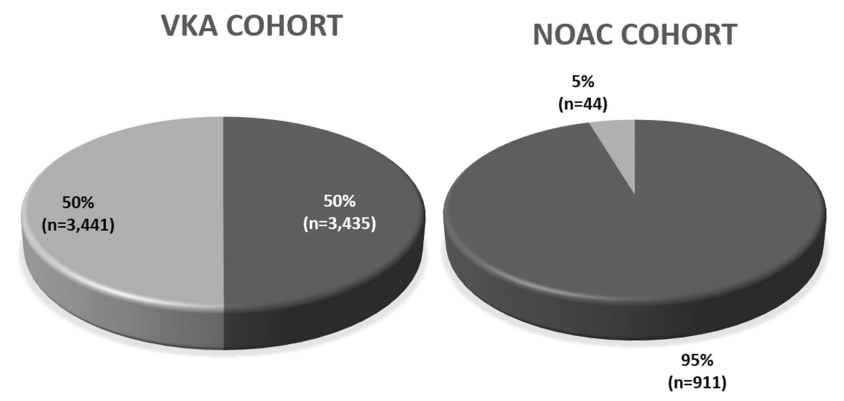

Figure 2 The distribution of the study population stratified by treatment assignment I year before the index date (A: VKA cohort; B: NOAC cohort).
$2.5 \mathrm{mg}$ for apixaban) were similar, both for naïve user and for OAC-experienced users.

The baseline characteristics of the VKA-naïve users and NOAC-naïve users are shown in Table 1.

At baseline, the mean age ranged from 75.4 years in patients newly treated with VKA to 77.4 years in patients newly treated with NOAC $(\mathrm{p}<0.001)$.

Patients newly treated with VKA had higher rate of previous hospitalisation for chronic kidney disease $(6.4 \%$ compared to $3.1 \%$ for NOAC-naïve users, $\mathrm{p}<0.001$ ), heart failure ( $25.9 \%$ compared to $16.6 \%$ for NOAC-naïve users, $\mathrm{p}<0.001$ ), coronary artery disease $(8.1 \%$ compared to $5.4 \%$ for NOACnaïve users, $\mathrm{p}=0.005)$ and myocardial infarction $(6.1 \%$ compared to $3.5 \%$ for NOAC-naïve users, $\mathrm{p}=0.002$ ) compared to NOAC-naïve users (Table 1). Whereas, newly treated with NOAC patients had a higher rate of previous hospitalisation for stroke (25.9\% compared to $13.7 \%$ for VKA naïve users, $\mathrm{p}<0.001$ ) (Table 1).

Newly treated with VKA patients more likely received previous treatment with other antihypertensive (57.5\% compared to $63.4 \%$ for NOAC-naïve users), less likely received a previous treatment with beta-blockers $(41.5 \%$ compared to $46.3 \%$ for NOAC-naïve users, $\mathrm{p}=0.009$ ) and had a higher average number of hospitalisation observed (1.4 compared to 1.3 for NOAC-naïve users, $\mathrm{p}<0.001$ ) (Table 1).

The data on comorbidity profile, bleeding and stroke risk at the baseline are also shown in Table 1.

At baseline, the use of antiplatelet drugs among VKAnaïve users and NOAC-naïve users resulted similar (46.9\% for VKA and $46.8 \%$ for NOACs) (Figure 3). During the followup period, the use of antiplatelet drugs decreased in both groups $(27.5 \%$ for VKA and $14.1 \%$ for NOACs).

Figure 4 show adherence data for the NOAC users, calculated during the follow-up period. Among patients who received NOAC at ID, 76.1\% showed an adherence level $\geq 80$ and $9.1 \%$ had an adherence level lower than $40 \%$.

The data on bleeding incidence events during the followup period are shown in Table 2. The unadjusted incidence rate for bleeding events was 10.46 per 1,000 person-year for VKA patients and 4.55 per 1000 person-years for NOAC patients. The unadjusted incidence rate for gastrointestinal bleeding was 4.98 per 1,000 person-years for VKA patients and 3.41 per 1,000 person-years for NOAC patients. The unadjusted incidence rate of death was 8.69 per 1,000 person-years for VKA patients and 8.61 per 1,000 person-years for NOAC patients. 
Table I Baseline Characteristics Among VKA And- NOAC-Naïve Users, Stratification According To Therapies Used During Pre-ID Period

\begin{tabular}{|c|c|c|c|}
\hline Baseline Characteristics* & VKA-Naïve Users, OAC Naïve & NOAC-Naïve Users, OAC Naïve & p Value \\
\hline Patients n (\%) & 3,435 & 911 & \\
\hline Age, years mean (SD) & $75.4(10.6)$ & $77.4(10.2)$ & $<0.001$ \\
\hline Men, n (\%) & 53.8 & 49.7 & 0.029 \\
\hline \multicolumn{4}{|l|}{ Comorbidities, (\%) } \\
\hline Hypertension & 19.5 & 18.7 & 0.579 \\
\hline Chronic kidney disease & 6.4 & 3.1 & $<0.001$ \\
\hline Coronary artery disease & 8.1 & 5.4 & 0.005 \\
\hline Heart failure & 25.9 & 16.6 & $<0.001$ \\
\hline Stroke & 13.7 & 25.9 & $<0.001$ \\
\hline Intracranial hemorrhage & 0.5 & 0.4 & 0.916 \\
\hline Major bleeding & 1.7 & 1.8 & 0.936 \\
\hline Myocardial infarction & 6.1 & 3.5 & 0.002 \\
\hline \multicolumn{4}{|l|}{ Medication use, (\%) } \\
\hline Betablockers & 41.5 & 46.3 & 0.009 \\
\hline Calcium channel blockers & 27.4 & 25.9 & 0.359 \\
\hline Diuretics & 32.4 & 31.7 & 0.685 \\
\hline Other antihypertensive & 57.5 & 63.4 & 0.001 \\
\hline \multicolumn{4}{|l|}{ Measures of overall health status } \\
\hline Number of unique medications, mean (SD) & $4.3(2.1)$ & $4.4(1.9)$ & 0.168 \\
\hline Number of hospitalisations observed, mean (SD) & $\mathrm{I} .4(\mathrm{I} .2)$ & $\mathrm{I} .3(\mathrm{I} .0)$ & $<0.001$ \\
\hline Number of physician visits observed, mean (SD) & $3.0(3.8)$ & $2.8(3.4)$ & 0.058 \\
\hline Charlson index, (\%) & & & 0.179 \\
\hline Low $(0-1)$ & 53.9 & 53.3 & \\
\hline Intermediate $(2-3)$ & 36.5 & 38.9 & \\
\hline High $(\geq 4)$ & 9.5 & 7.8 & \\
\hline HAS BLED, (\%) & & & 0.007 \\
\hline Low $(0-1)$ & 17.9 & 13.6 & \\
\hline Intermediate (2) & 27.8 & 28.0 & \\
\hline High $(\geq 3)$ & 54.3 & 58.4 & \\
\hline CHA2Ds2 VASc, (\%) & & & $<0.001$ \\
\hline Low (0-3) & 47.9 & 41.7 & \\
\hline Intermediate (4) & 25.7 & 25.0 & \\
\hline High $(\geq 5)$ & 26.3 & 33.3 & \\
\hline
\end{tabular}

Notes: *Evaluated in the I year prior to the index date.

Health care resource consumption (in term of drugs, hospitalisations and outpatient specialist services) and related costs among adult patients with at least one prescription of VKA or NOAC enrolled from 1 July 2013 to 30 June 2015 were explored according to their treatment at the ID (Figure 5). Of our study population, 1,009 patients were followed-up for at least 12 months; on these patients, the cost analysis was performed. On average, the overall costs were $€ 5,156.13$ for VKA patients and $€ 4,630.57$ for NOAC patients.

\section{Discussion}

The purpose of the study was to evaluate anticoagulation treatment patterns and health care resource use in adult patients with a diagnosis of NVAF and to describe the characteristics of patients affected by NVAF in relation to treatment.

The analyses were done retrospectively in unselected Italian patient population in routine clinical practice. In this real-world setting, of the 32,863 patients who were diagnosed with NVAF, $23.8 \%(n=7,831)$ received a 


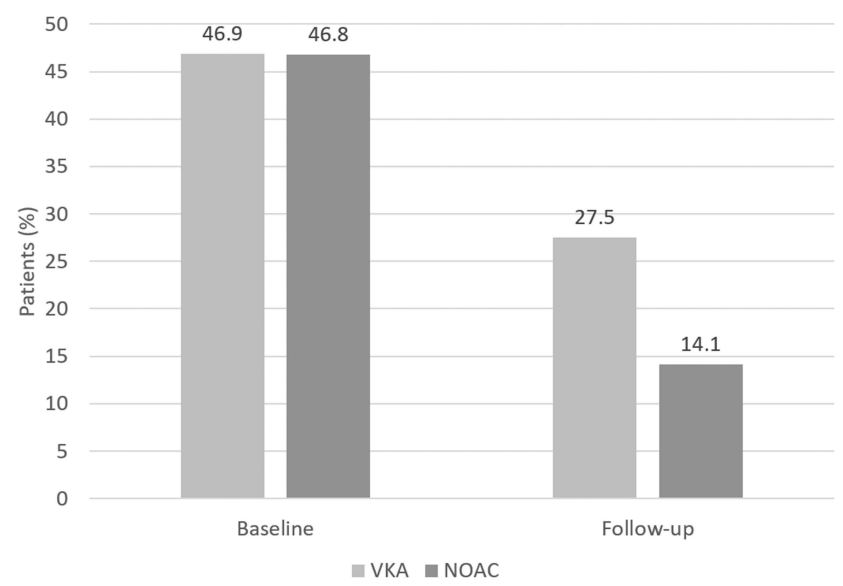

Figure 3 Use of antiplatelet agents among OAC-naïve users during the baseline and follow-up period.

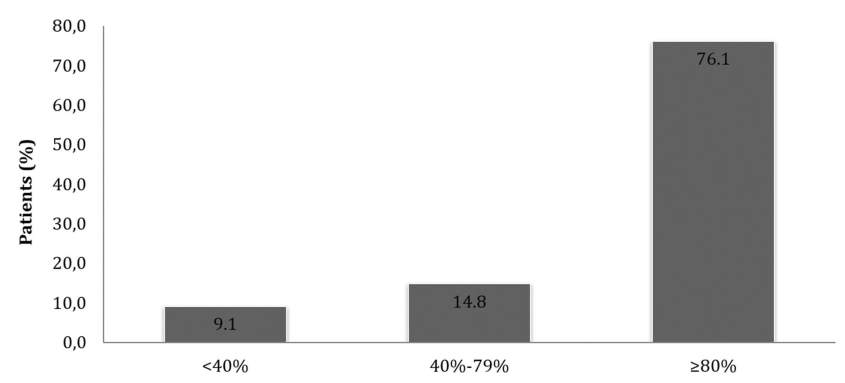

Figure 4 Adherence to NOAC treatment during the first 12 months after the index-date.

prescription of oral anticoagulant; about $87.8 \%$ of them were prescribed VKA and $12.2 \%$ were treated with NOACs. Results among NVAF patients receiving OACs are consistent with other Italian studies, which showed higher antiplatelet drug prescription compared to OACs $;{ }^{11,12}$ moreover, VKAs were still largely prescribed. Patients naïve to oral anticoagulants who were prescribed NOAC instead of VKA may be considered a new group

Table 2 Incidence Of Bleeding Events

\begin{tabular}{|l|l|l|}
\hline & VKA (N) & NOAC (N) \\
\hline Patients & 6,155 & 927 \\
Patients with events (\%) & $196(3.2)$ & $4(0.4)$ \\
Events & 229 & 5 \\
Person-years of follow-up & 18,740 & 878 \\
Incidence rate/I,000-person years & 10.46 & 4.55 \\
Incidence rate of Gl bleeding events/ & 4.98 & 3.41 \\
I,000-person years & & \\
\hline
\end{tabular}

Notes: Analysis made on 7082 patients with the same class during the follow-up period. Bleeding events have been evaluated during the follow-up period.

Abbreviations: VKA, vitamin $\mathrm{K}$ antagonist; NOAC, non-vitamin $\mathrm{K}$ oral anticoagulant agents; GI, gastrointestinal.

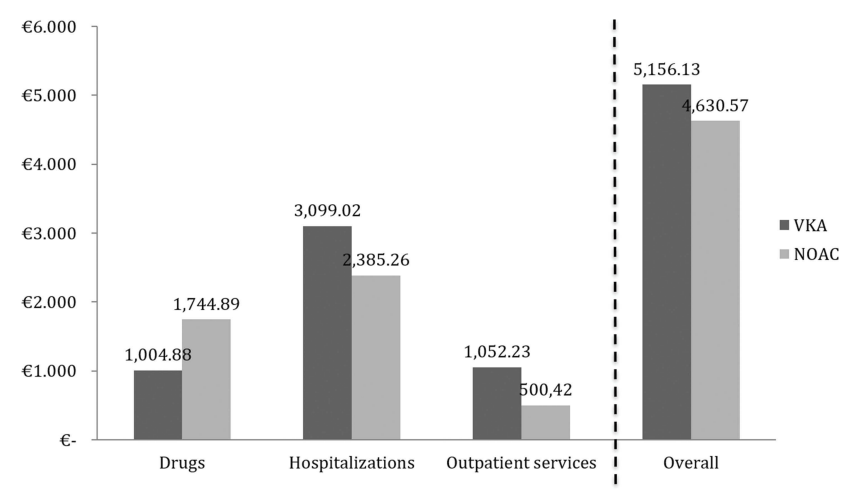

Figure 5 Total cost during the I-year after the index-date.

of patients since VKA has previously been the usual standard of care and is cheaper to prescribe than the NOACs. $^{13}$

Our findings showed that the majority of NOAC users were patients newly treated with OACs, and only about $5 \%$ of the NOAC users had been previously treated with VKA.

Trends in the prescription of novel oral anticoagulants into different countries were examined. Kirley $\mathrm{K}$ et $\mathrm{al}^{14}$ have conducted a study to examine overall OAC utilisation between 2007 and 2011 in the USA, with a specific focus on dabigatran and warfarin. The study findings demonstrated that dabigatran has been rapidly adopted into ambulatory practice in the USA, but this study did not provide evidence that NOAC utilisation has increased overall NVAF treatment rates. However, a further investigation on the same database showed a trend in increasing OAC prescriptions among patients with atrial fibrillation. ${ }^{15}$

In our examination of the patient characteristics across the OAC-naïve cohorts, we found that $25.9 \%$ of the NOAC (newly treated) cohort and $13.7 \%$ in the VKA (newly treated) cohort had a history of stroke. Our observational data were in accordance with the recent report from England, where history of stroke and bleeding was the highest among apixaban (23.7\% and $31.6 \%)$ and lowest among VKA patients (15.9\% and $27.5 \%) .{ }^{13}$ In addition, this aspect could be explained by the fact that apixaban has the lowest rate of intracerebral bleeding, as shown by the ARISTOTLE study. ${ }^{4}$

We also found that VKA tended to be prescribed in patients with previous hospitalisation for heart failure (25.9\% vs $16.6 \%$ for NOAC users, p <0.001), probably because a proportion of them was affected by an impaired renal function. In addition to that, the higher use of VKA in patients with previous hospitalisation for coronary artery disease $(8.1 \%$ vs $5.4 \%$ for NOAC users, $\mathrm{p}=0.005)$ 
and for myocardial infarction $(6.1 \%$ vs $3.5 \%$ for NOAC users, $\mathrm{p}=0.002$ ) may possibly reflect the perceived feeling that warfarin provides better protection against coronary ischemic events than NOACs, ${ }^{16-18}$ but the explanation for this finding is therefore uncertain.

A consistent proportion of patients (around 47\%) still received antiplatelet agent in OAC-naïve user cohort. This result may imply that doctors still consider antiplatelet agent safer than OAC (either warfarin or NOACs) in a clinical setting. Our findings are not dissimilar from those of other studies in practice-based circumstances published so far. ${ }^{13,18}$ The results showing a higher use of antiplatelet agent among VKA-naïve users than the NOAC-naïve users should be discussed by considering previous hospitalisation for coronary artery disease. In routine clinical practice, many clinicians are still confident in prescribing a combination of warfarin plus aspirin in patients with stable coronary artery disease and atrial fibrillation, despite the guidelines recommending the monotherapy with warfarin (or a NOAC). ${ }^{19}$

Recently, analyses of registry data have shown that there is still much room for improvement in the use of anticoagulants in routine clinical practice. ${ }^{20-23}$ This retrospective analysis highlights that a high proportion of patients tend to be prescribed the lower dose of NOACs. Results from Phase III studies suggested that the proportion of patients prescribed low-dose NOACs was $49.7 \%$ for dabigatran $110 \mathrm{mg}$ twice a day, $20.7 \%$ for rivaroxaban $15 \mathrm{mg}$ once a day and $4.7 \%$ for apixaban $2.5 \mathrm{mg}$ twice a day; however, real-world data have shown that the proportions of patients prescribed low-dose NOACs was much higher$53.7 \%, 24.5 \%$ and $35.5 \%$ for dabigatran, rivaroxaban and apixaban, respectively. ${ }^{24,25}$ The available literature showing reduced dose regimens of NOACs from "real-world" clinical practice is little. Recently, Nielsen et $\mathrm{al}^{24}$ investigated the effectiveness and safety of low-dose NOAC regimens compared with warfarin in a large unselected cohort of Danish patients with atrial fibrillation who had not undetgone treatment with OACs. They found that, rates of ischemic stroke/systemic embolism with apixaban $2.5 \mathrm{mg}$ twice a day were higher but not significantly so compared with warfarin, whereas dabigatran $110 \mathrm{mg}$ twice a day and rivaroxaban $15 \mathrm{mg}$ once a day both showed a trend towards lower thromboembolic rates. Rates of bleeding were not significantly different for apixaban and rivaroxaban compared with warfarin, but they were significantly lower for dabigatran. $^{25}$

Treatment adherence is commonly studied using routinely collected data, such as those used in this study. ${ }^{26}$ Our data indicated that among NOAC users, $76.1 \%$ of them showed an adherence level $\geq 80 \%$. Despite methodological differences, our result on high treatment adherence with NOACs does not differ from findings of other studies that used different methodological approaches. ${ }^{27}$ High adherence level with NOAC could be expected because NOACs do not require routine monitoring and do not have food-drug interactions and fewer drug-drug interactions. Our findings of high adherence levels with NOAC may be explained by the improved convenience of these modern therapies. ${ }^{3,20}$ Considering that the clinical use of NOAC drugs is actually regulated in Italy by bureaucratic restrictions, clinical prescription of NOACs preliminarily requires an online prescription plan which should be compiled on the Italian Drug Agency website. ${ }^{28}$

Among NVAF patients in the present real-world setting, the percentage of patients with major bleeding was lower in NOAC patients compared to VKA. Although this study is consistent with others showing a decreased bleeding risk with NOACs compared with warfarin, ${ }^{29,30}$ the small number of bleeding episodes in NOAC users limited the statistical power to confirm the reductions in bleeding risk. Hopefully, future prospective with a larger cohort of NOAC patients and with a control for potential confounders will allow comparisons between these drugs for multiple clinical outcomes. Nevertheless, it is well known that bleeding complications during the OAC treatment are associated with a higher rates of hospitalisations and related costs. ${ }^{29,30}$

In accordance with data observed in other studies, ${ }^{31-34}$ based on real-world data, our results highlight that when any of the evaluated NOACs are used instead of warfarin for treatment of patients with NVAF, annual medical costs are reduced.

Our results should be interpreted in light of potential limitations. Our cohort of patients reflected real clinical practice, and the results must be interpreted, taking into account limitations related to the observational nature of the study, based on data collected through administrative and laboratory databases. First, proxy has been used to evaluate comorbidities and severity of illness, as clinical data were not retrievable from our dataset. Second, data on patients' exposure to pharmacological treatment were acquired from medical prescription and dispensing; thus, no information on actual drug use was available. Third, INR values for patients on warfarin were not available, along with dose adjustment; thus, data on treatment adherence were not presented for these patients and costs were analysed assuming a standard dosage, already used in a previous study. Finally, our sample included a small proportion of the Italian health-assisted individuals diagnosed with 
NAVF and newly treated with OACs, which limits the generalisation of our results to the whole population with NAVF treated with OACs.

The main strength of the present study is the nature of the databases used: administrative databases represent a suitable source of health care data, as they comprise almost all the information on services provided by the Italian National Health Service.

This unselected cohort study, in an Italian setting, on NVAF patients being prescribed OAC highlights that VKA was largely prescribed, and the great majority of patients treated with NOAC were adherent to treatment. Most of the patients still received antiplatelet agent for prevention. In addition, in NOAC patients, we registered lower bleeding events compared to VKA patients. A larger study with longer follow-up of NOAC users is needed to support these findings. The results and conclusions of this study are limited to the population analysed.

Evidence from real-world studies can provide answers to questions important for both payers and providers, such as quality of care and value of provision. Health care providers consider comparative effectiveness and safety information, particularly from real-world use, an important tool in making treatment decisions at the point of care. Payers need practicebased evidence to make formulary and reimbursement decisions and to understand how to best incorporate new therapies and technologies into everyday clinical practice.

\section{Strengths And Limitations Of This Study}

- This study, in a "real-world" setting, is one of only a few studies investigating the current use of NVAF treatments in an Italian practice-based perspective.

- Administrative databases analysed in the present study represent a suitable source of health care data, as they comprise almost all the information on services provided by the Italian National Health Service.

- The sample size was relatively limited, and although we used two health care databases comprising a total of approximately two million individuals who are assisted by the National Health System in two regions of Italy, larger studies are needed to confirm and to enhance the generalisability of the findings, and in different populations.

- Some limitations, characteristics of retrospective observational study on administrative databases should be declared. First, clinical data were not retrievable from the dataset. Second, data on patients' pharmacological treatment exposure were acquired from medical prescriptions and dispensing. Third, INR values and dose adjustment were not available for patients on warfarin; thus, data on treatment adherence were not presented and costs were analysed assuming a standard dosage.

\section{Ethics Approval}

For this type of study, formal consent is not required. However, to guarantee patient privacy, no personal identifiers were provided to the researchers. According to the Italian law for confidentiality of data, the study was notified to the Ethics Committees of each local health unit.

\section{Data Sharing Statement}

Data are not available due to ethical issues.

\section{Author Contributors}

All authors contributed to data analysis, drafting or revising the article, gave final approval of the version to be published and agree to be accountable for all aspects of the work.

\section{Disclosure}

All authors declare no support from any organisation for the submitted work.

\section{References}

1. Kirchhof P, Benussi S, Kotecha D, et al. 2016 ESC Guidelines for the management of atrial fibrillation developed in collaboration with EACTS. Eur Heart J. 2016;37:2893-2962.

2. Wolf PA, Abbott RD, Kannel WB. Atrial fibrillation as an independent risk factor for stroke: the Framingham Study. Stroke. 1991;22:983988. doi:10.1161/01.STR.22.8.983

3. Heidbuchel H, Verhamme P, Alings M, et al. Updated European Heart Rhythm Association Practical Guide on the use of non-vitamin K antagonist anticoagulants in patients with non-valvular atrial fibrillation. Europace. 2015;17:1467-1507. doi:10.1093/europace/euv309

4. Granger CB, Alexander JH, McMurray JJ, et al. Apixaban versus warfarin in patients with atrial fibrillation. $N$ Engl $J$ Med. 2011;365:981-992. doi:10.1056/NEJMoa0910383

5. Connolly SJ, Ezekowitz MD, Yusuf S, et al. Dabigatran versus warfarin in patients with atrial fibrillation. N Engl J Med. 2009;361:11391151. doi:10.1056/NEJMoa0905561

6. Giugliano RP, Ruff CT, Braunwald E, et al. Edoxaban versus warfarin in patients with atrial fibrillation. N Engl J Med. 2013;369:2093-2104. doi:10.1056/NEJMoa1310907

7. Patel MR, Mahaffey KW, Garg J, et al. Rivaroxaban versus warfarin in nonvalvular atrial fibrillation. $N$ Engl J Med. 2011;365:883-891. doi:10.1056/NEJMoa0910383 
8. Degli Esposti L, Sangiorgi D, Di Pasquale G, Gensini GF, Iori I, Buda S, Degli Esposti E; on the behalf of the Study Group. Adherence to treatment and anticoagulation control in vitamin $\mathrm{K}$ antagonists-treated patients: an administrative databases analysis in a large Italian population. Farmeconomia. 2011;12(2):53-59.

9. Larsen TB, Skjøth F, Nielsen PB, Kjældgaard JN, Lip GYH. Comparative effectiveness and safety of non-vitamin $\mathrm{K}$ antagonist oral anticoagulants and warfarin in patients with atrial fibrillation: propensity weighted nationwide cohort study. BMJ. 2016;13189. doi:10.1136/bmj.i3189

10. Opinion 05/2014 on Privacy and Data Protection. Available from: http:// ec.europa.eu/justice/data-protection/article-29/documentation/opinionrecommendation/files/2014/wp216 it.pdf. Accessed September 27, 2019.

11. Cataldo N, Pegoraro V, Ripellino C, et al. Non-persistence risk and health care resource utilization of Italian patients with non-valvular atrial fibrillation. Recenti Prog Med. 2018. doi:10.1701/2865.28904

12. Citarella A, Guida A, Capogrosso P, et al. Scelta della terapia antitrombotica in pazienti con fibrillazione atriale non valvolare nella reale pratica clinica. Recenti Prog Med. 2016. doi:10.1701/2260.24336

13. Johnson ME, Lefèvre C, Collings S-L, et al. Early real-world evidence of persistence on oral anticoagulants for stroke prevention in non-valvular atrial fibrillation: a cohort study in UK primary care. BMJ Open. 2016;6:e011471. doi:10.1136/bmjopen-2016-011471

14. Kirley K, Qato DM, Kornfield R, Stafford RS, Alexander GC National trends in oral anticoagulant use in the United States, 2007 to 2011. Circ Cardiovasc Qual Outcomes. 2012;5:615-621. doi:10.1161/CIRCOUTCOMES.112.967299

15. Barnes GD, Lucas E, Alexander GC, Goldberger ZD. National trends in ambulatory oral anticoagulant use. Am J Med. 2015;128:13001305.e2. doi:10.1016/j.amjmed.2015.05.044

16. Giglio AF, Basile E, Santangeli P, Di Biase L, Trotta F, Natale A. Increased risk of myocardial infarction with dabigatran: fact or fiction? J Cardiovasc Med. 2014;15:19-26. doi:10.2459/JCM.0b013e328364beb8

17. Ezekowitz MD, Connolly S, Parekh A, et al. Rationale and design of RE-LY: randomized evaluation of long-term anticoagulant therapy, warfarin, compared with dabigatran. Am Heart J. 2009;157:805-810. e2. doi:10.1016/j.ahj.2009.02.005

18. Kumar R, Rahman AM, Henry BL. A review of the clinical subgroup analyses from the RE-LY trial. Rev Cardiovasc Med. 2016;17:40-48.

19. Lin Y-P, Tan T-Y. Do NOACs improve antithrombotic therapy in secondary stroke prevention in nonvalvular atrial fibrillation? Medicine (Baltimore). 2015;94:e1627. doi:10.1097/MD.0000000000000874

20. Ten Cate V, Ten Cate H, Verheugt FWA. The global anticoagulant registry in the FIELD-atrial fibrillation (GARFIELD-AF): exploring the changes in anticoagulant practice in patients with non-valvular atrial fibrillation in the Netherlands. Neth Heart J. 2016;24:574-580. doi:10.1007/s12471-016-0874-y

21. Huisman MV, Rothman KJ, Paquette M, et al. The changing landscape for stroke prevention in AF. J Am Coll Cardiol. 2017;69:777785. doi:10.1016/j.jacc.2016.11.026

22. Kirchhof P, Ammentorp B, Darius H, et al. Management of atrial fibrillation in seven European countries after the publication of the 2010 ESC Guidelines on atrial fibrillation: primary results of the PREvention oF thromboemolic events - european Registry in Atrial Fibrillation (PREFER in AF). EP Eur. 2014;16:6-14.
23. Proietti M, Laroche C, Opolski G, et al. 'Real-world' atrial fibrillation management in Europe: observations from the 2-year follow-up of the EURObservational research programme-atrial fibrillation general registry pilot phase. Europace Euw. 2016;112. doi:10.1093/europace/euw112

24. BJCardio Staff. Anticoagulation highlights from the ESC. $\mathrm{Br} J$ Cardiol. 2016;23(4).

25. Nielsen PB, Skjøth F, Søgaard M, Kjældgaard JN, Lip GY, Larsen TB. Effectiveness and safety of reduced dose non-vitamin K antagonist oral anticoagulants and warfarin in patients with atrial fibrillation: propensity weighted nationwide cohort study. BMJ. 2017;j510. doi:10.1136/bmj.j510

26. Agenzia Italiana del Farmaco (AIFA). L'uso dei farmaci in Italiarapporto OsMed 2015. Available from: http://www.agenziafar maco.gov.it/sites/default/files/Rapporto_OsMed2015.pdf. Accessed September 27, 2019.

27. Beyer-Westendorf J, Ehlken B, Evers T. Real-world persistence and adherence to oral anticoagulation for stroke risk reduction in patients with atrial fibrillation. Europace. 2016;18:1150-1157. doi:10.1093/ europace/euv421

28. Agenzia Italiana del Farmaco (AIFA). AIFA Concept paper. I nuovi anticoagulanti orali nella prevenzione di ictus e tromboembolismo sistemico in pazienti con fibrillazione atriale non valvolare. Available from: http://www.agenziafarmaco.gov.it/sites/default/files/version 2012_09_24_cp_noacs_1.pdf. Accessed September 27, 2019.

29. Cangemi DJ, Krill T, Weideman R, Cipher DJ, Spechler SJ, Feagins LA. A comparison of the rate of gastrointestinal bleeding in patients taking non-vitamin $\mathrm{K}$ antagonist oral anticoagulants or warfarin. Am J Gastroenterol. 2017;112:734-739. doi:10.1038/ ajg.2017.39

30. Verdecchia P, Angeli F, Aita A, Bartolini C, Reboldi G. Why switch from warfarin to NOACs? Intern Emerg Med. 2016;11:289-293. doi:10.1007/s11739-016-1411-0

31. Amin A, Bruno A, Trocio J, Lin J, Lingohr-Smith M. Comparison of differences in medical costs when new oral anticoagulants are used for the treatment of patients with non-valvular atrial fibrillation and venous thromboembolism vs warfarin or placebo in the US. J Med Econ. 2015;18:399-409. doi:10.3111/13696998.201 5.1007210

32. Amin A, Stokes M, Makenbaeva D, Wiederkehr D, Wu N, Lawrence JH. Estimated medical cost reductions associated with use of novel oral anticoagulants vs warfarin in a real-world non-valvular atrial fibrillation patient population. $J$ Med Econ. 2014;17:771-781. doi:10.3111/13696998.2014.953682

33. Urooj F, Kulkarni A, Stapleton D, Kaluski E. New oral anticoagulants in nonvalvular atrial fibrillation: new oral anticoagulants in nonvalvular atrial fibrillation. Clin Cardiol. 2016;39:739-746. doi:10.1002/clc.2016.39.issue-12

34. Deitelzweig S, Amin A, Jing Y, et al. Medical cost reductions associated with the usage of novel oral anticoagulants vs warfarin among atrial fibrillation patients, based on the RE-LY, ROCKET-AF, and ARISTOTLE trials. J Med Econ. 2012;15:776-785. doi:10.3111/ 13696998.2012.680555
Vascular Health and Risk Management

\section{Publish your work in this journal}

Vascular Health and Risk Management is an international, peerreviewed journal of therapeutics and risk management, focusing on concise rapid reporting of clinical studies on the processes involved in the maintenance of vascular health; the monitoring, prevention and treatment of vascular disease and its sequelae; and the involvement of metabolic disorders, particularly diabetes. This journal is indexed on PubMed Central and MedLine. The manuscript management system is completely online and includes a very quick and fair peerreview system, which is all easy to use. Visit http://www.dovepress. com/testimonials.php to read real quotes from published authors. 